\title{
Function of the MAPK scaffold protein, Ste5, requires a cryptic $\mathrm{PH}$ domain
}

\author{
Lindsay S. Garrenton, Susan L. Young, and Jeremy Thorner ${ }^{1}$ \\ Department of Molecular and Cell Biology, Division of Biochemistry and Molecular Biology, University of California, \\ Berkeley, California 94720, USA
}

\begin{abstract}
Ste5, the prototypic mitogen-activated protein kinase (MAPK) scaffold protein, associates with plasma membrane-tethered G $\beta \gamma$ freed upon pheromone receptor occupancy, thereby initiating downstream signaling. We demonstrate that this interaction and membrane binding of an N-terminal amphipathic $\alpha$-helix (PM motif) are not sufficient for Ste5 action. Rather, Ste5 contains a pleckstrin-homology (PH) domain (residues 388-518) that is essential for its membrane recruitment and function. Altering residues (R407S K411S) equivalent to those that mediate phosphoinositide binding in other PH domains abolishes Ste5 function. The isolated PH domain, but not a R407S K411S derivative, binds phosphoinositides in vitro. Ste5(R407S K411S) is expressed normally, retains G $\beta \gamma$ and Ste11 binding, and oligomerizes, yet is not recruited to the membrane in response to pheromone. Artificial membrane tethering of Ste5(R407S K411S) restores signaling. R407S K411S loss-of-function mutations abrogate the constitutive activity of gain-of-function Ste5 alleles, including one (P44L) that increases membrane affinity of the PM motif. Thus, the PH domain is essential for stable membrane recruitment of Ste5, and this association is critical for initiation of downstream signaling because it allows Ste5-bound Ste11 (MAPKKK) to be activated by membrane-bound Ste20 (MAPKKKK).
\end{abstract}

[Keywords: Pheromone response; plasma membrane; mutants; yeast; Saccharomyces cerevisiae; Ste20]

Supplemental material is available at http://www.genesdev.org.

Received January 26, 2006; revised version accepted May 8, 2006.

Yeast pheromone response links activation of a G-protein-coupled receptor to stimulation of an appropriate mitogen-activated protein kinase (MAPK) (for review, see Wang and Dohlman 2004). Ste5 protein (Elion 2001) is essential for this coupling: First, because it binds the MAPKKK (Ste11), the MAPKK (Ste7), and the MAPK (Fus3) of the MAPK cascade. Distinct regions of Ste5 interact with each kinase, as delimited by mutational analysis (Choi et al. 1994; Marcus et al. 1994; Printen and Sprague 1994; Inouye et al. 1997a); thus, Ste5 was the first recognized MAPK scaffold protein. Second, Ste5 delivers its kinase cargo to the plasma membrane, at least in part, via association with the G $\beta$ subunit (Ste4) of the G $\beta \gamma$ complex released from the receptor-associated heterotrimeric G-protein (Whiteway et al. 1995; Pryciak and Huntress 1998). The region of Ste5 responsible for interaction with G $\beta \gamma$ includes a RING-H2 domain near its $\mathrm{N}$ terminus (Inouye et al. 1997b; Feng et al. 1998). G $\beta \gamma$ remains tethered at the plasma membrane via lipophilic modifications at the C-terminal end of $\mathrm{G} \gamma$ (Ste18) (Hirschman and Jenness 1999). G $\beta \gamma$-dependent docking of Ste5 at the plasma membrane permits efficient encounter of Ste5-bound Ste11 with its activator

${ }^{1}$ Corresponding author.

E-MAIL jthorner@berkeley.edu; FAX (510) 642-6420.

Article is online at http://www.genesdev.org/cgi/doi/10.1101/gad.1413706.
(MAPKKKK), Ste20 (Drogen et al. 2000), a so-called p21activated protein kinase (PAK). The $\mathrm{p} 21$ that activates Ste20 is the small GTPase, Cdc42 (Lamson et al. 2002), which is also membrane-anchored via C-terminal lipophilic modifications (Ohya et al. 1993). Activated Ste20 is membrane-associated not only by binding of its $\mathrm{N}$ terminal CRIB domain to Cdc42-GTP, but also because its $\mathrm{C}$ terminus contains a high-affinity binding site for Gßy (Leeuw et al. 1998). Thus, like Ste5, Ste20 concentrates near activated pheromone receptors.

Ste 5 self-associates, and this oligomerization is important for efficient signaling (Yablonski et al. 1996; Inouye et al. 1997b; Wang and Elion 2003). Ste5 undergoes constitutive nucleocytoplasmic shuttling in naïve cells, but accumulates stably at the cell cortex in the projection ("shmoo tip") that forms on pheromone-treated cells (Pryciak and Huntress 1998; Mahanty et al. 1999), and reimport of Ste5 into the nucleus contributes to downregulation of signaling (Künzler et al. 2001). To further delineate how Ste5 contributes to signal propagation, we isolated three mutations (P44L, C226Y, S770N) that constitutively activate Ste5 (Sette et al. 2000) by selecting for alterations of Ste5 that permit signaling even in cells lacking G $\beta \gamma$. Although these alleles bypass the need for $\mathrm{G} \beta \gamma$, they do not bypass the need for the other known components of the pathway, most notably Ste20. Our ability to isolate such alleles raised a paradox, given the 
evidence that Ste5-Gßy association is critical for membrane delivery of Ste11 and its activation by Ste20. This quandary suggested that the mutants unveil an alternative, relatively efficient, G $\beta \gamma$-independent means by which Ste 5 associates with the membrane.

Perhaps the mutants are secured at the cell cortex, even in the absence of G $\beta \gamma$, because they enhance Ste5 association with other proteins that are themselves membrane-bound in a G $\beta \gamma$-independent manner. Interactions of Ste 5 with other plasma membrane-associated proteins, including Bem1 (Leeuw et al. 1995; Lyons et al. 1996) and, most recently, Cdc24 (Wang et al. 2005), have been reported. Bem1, an SH3 domain-containing adaptor protein, has an internal Phox-homology (PX) domain, and PX domains can bind phosphoinositides (Sato et al. 2001). Cdc24, the guanine nucleotide exchange factor (GEF) for Cdc42, has an internal pleckstrin-homology (PH) domain, and $\mathrm{PH}$ domains can also bind phosphoinositides (for review, see Lemmon and Keleti 2005).

Another possibility is that the constitutive alleles elevate some intrinsic ability of Ste5 to associate with membranes. Indeed, it has been demonstrated that P44L (and T52M, another constitutive Ste5 allele that we isolated by a different strategy [Hasson et al. 1994]) operate by enhancing the membrane-binding affinity of an $\mathrm{N}$ terminal amphipathic $\alpha$-helix in Ste5, dubbed the PM motif (Winters et al. 2005). However, some sequence analysis algorithms predict that Ste 5 contains a PH domain. We suspected that the candidate $\mathrm{PH}$ domain might be highly relevant to Ste5 function because some PH domains can associate with both phosphoinositides and GBy (Lemmon and Keleti 2005). Recent genome-wide analysis of Saccharomyces cerevisiae PH domains relied on the SMART database, which did not pinpoint Ste5 (Yu et al. 2004). Hence, the properties and biological role of the presumptive PH domain in Ste5 have not been explored. Therefore, we applied genetic, biochemical, and cell biological approaches to assess the role of the predicted PH domain in Ste5. As documented here, this previously unrecognized and unstudied region of Ste5 is absolutely essential for the function of Ste 5 in pheromone signaling because it is required to achieve stable membrane recruitment of Ste5.

\section{Results}

\section{A candidate PH domain in Ste5}

Both the PFAM (Bateman et al. 2004) and BLAST CDD (Marchler-Bauer et al. 2003) databases predict a PH domain (residues 400-512) in S. cerevisiae Ste5. This element lies in a slightly larger region (residues 388-518) that is highly conserved between S. cerevisiae Ste5 and its orthologs in other Saccharomyces species and even more distantly related yeasts (Fig. 1A). This segment possesses the sequence signatures (Fig. 1B) found in well characterized PH domains, even though PH domains in general can share as little as $10 \%-15 \%$ amino acid identity (Lemmon and Keleti 2005). In particular, the N-terminal portion of the presumptive PH domain in Ste5 contains basic residues situated at positions that are equivalent to those that mediate phosphoinositide binding and membrane targeting in crystal structures of other PH domains (Fig. 1B). Despite low sequence similarity, authentic PH domains all adopt a characteristic protein fold, consisting of a $\beta$-strand sandwich capped off by a C-terminal $\alpha$-helix. Regardless of the algorithm used (Pollastri et al. 2002; Lin et al. 2005), the apparent PH domain in Ste5 is predicted to assume the same tertiary fold (Fig. 1C), in agreement with its alignment against other $\mathrm{PH}$ domains of known structure based on sequence similarity alone (Fig. 1B). Moreover, molecular modeling and threading programs (Kelley et al. 2000; McGuffin et al. 2000) predict that, compared with the database of known folds, all those closest to the predicted structure of this region of Ste5 are PH domains.

\section{The predicted PH domain is essential for Ste5 function}

To investigate the role of this sequence in Ste 5 function in vivo, three mutants were generated: Ste5(R407S K411S); Ste5(K416S R420S); and Ste5(R407S K411S K416S R420S) (Fig. 2A). These basic residues were selected because they lie at or near positions in $\mathrm{PH}$ domains of known structure that contact the phosphates on the head group of the phosphoinositides to which they bind. When expressed at near-endogenous level (from the native STE5 promoter on a CEN plasmid), Ste5(R407S K411S) failed to rescue the mating defect of a ste $5 \Delta$ mutant, whereas Ste(K416S R420S) supported mating as well as wild-type Ste5 (Fig. 2B, left). The quadruple mutant displayed the same mating deficiency as Ste5(R407S K411S). The observed phenotypes were not due to any differences in expression or stability of these mutants, compared with each other or wild-type Ste5 (Fig. 2C, left). Nevertheless, partial loss-of-function mutations can sometimes be rescued by increased expression. Hence, as one means to assess the severity of the defect conferred by the R407S K411S mutations, the same test was performed with the same mutants overexpressed from the GAL1 promoter. Even when produced at a level 10-20 times above normal (Fig. 2C, right), both Ste5(R407S K411S) and the quadruple mutant were unable to support any detectable mating (Fig. $2 \mathrm{~B}$, right), indicating that the R407S K411S mutations confer a complete loss of function.

\section{Ste5 PH domain binds phosphoinositides in vitro}

A hallmark of many PH domains (but far from all) is the ability to bind specific phosphoinositides in vitro (Lemmon and Keleti 2005). To determine whether the Ste5 $\mathrm{PH}$ domain binds phosphoinositides and whether the phenotypes of the mutants correlate with their phosphoinositide-binding capability, we used three independent methods to measure the association of the isolated Ste5 PH domain with these lipids. For this purpose, we constructed, expressed, and purified from bacterial cells a glutathione-S-transferase (GST)-Ste5(369-517) fusion 


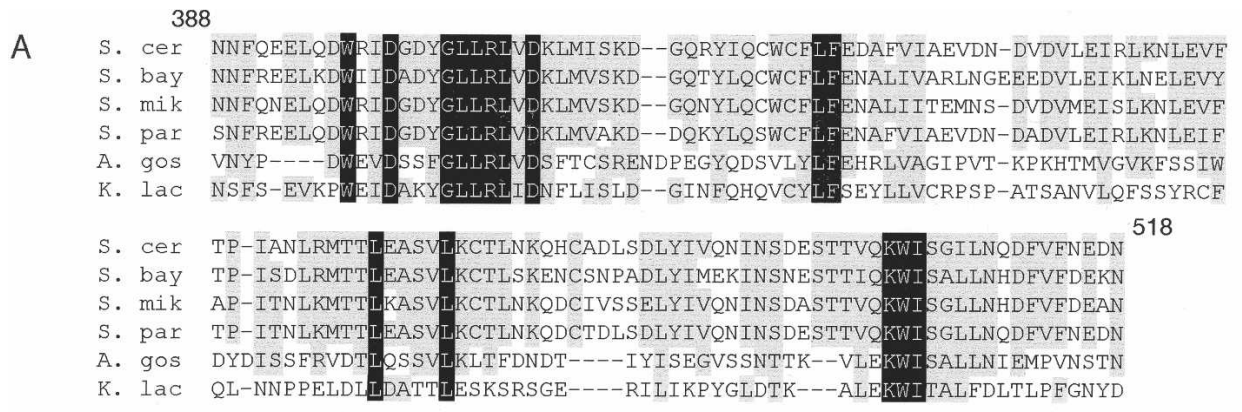

B
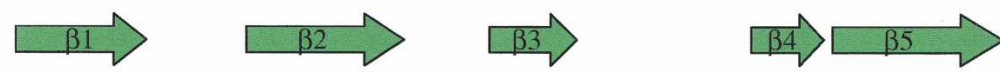

Ste 5: NNFQEELQDWRIDGDYGLLRLVDRLMISKDGQRYIQCWCELFEDAFVIAE-----VDNDVDVLEIRLKNLEVFT PIAN hPLC $\delta$-1: HGLQDDEDLQALIKGSQLL KVKSS---SWRRERFYKLQED-CKT IWQESRKV---MRT PESOLES IEDIOEVRMGHRT hGRK2: GHEEDYALGKDCIMHGYMSKMGNPELTQWQR-RYFYLF---PNRLEWRGE-----GEAPQ-SLLTMEEIQSVEETQIK hBTK: ---MAAVILESIFLKRSQQKKKTSPL-NEKK-RLFLLT---VHKLSYYEYDEERGRRGSKKGS IDVEKITCVETVVPE CNKSR2: RRI SCKDLGRGDCEGWLWKKRDARSYF SORWKKYWEVL---KDASYWYINEEDE--KAEGFISLPEEKIDRASECRKK

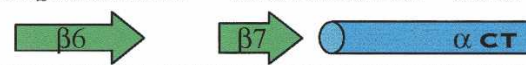

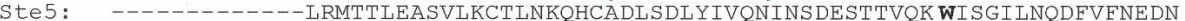
hPLC $\delta-1:----------E G L E K E A R D V P E D R C F S I V F K D Q R N T$ LDL IAPS PADAQH WVL GLHKI IHHSGSMD hGRK2: ----------------------ERKCLLLKIRGG-KQFILQCDSDPELVQ WKKELRDAYREAQQLV hBTK: KNPPPERQIPRRGEESSEMEQISIIERFPYPFQVVYDE--GPLYVFSPTEELRKR WIHQLKNVIRYNSDLV CNKSR2: - -

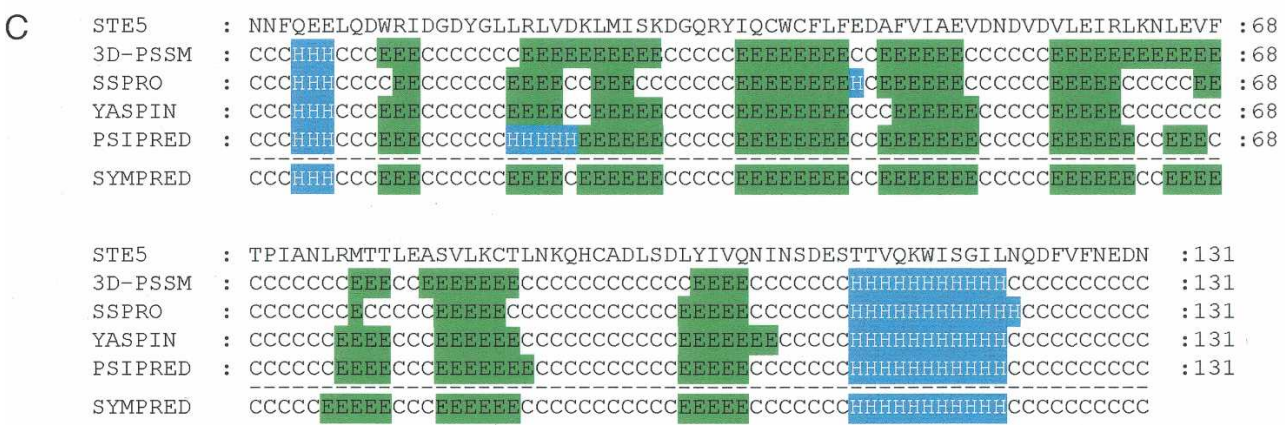

Figure 1. Sequence alignment and analysis of the predicted S. cerevisiae Ste5 PH domain. (A) Alignment of the S. cerevisiae Ste5 PH domain (residues 388-518) with corresponding sequences in orthologs from Saccharomyces bayanus, Saccharomyces mikatae, Saccharomyces paradoxus, Ashbya gossypii, and Kluyveromyces lactis. (B) Alignment of the S. cerevisiae Ste5 PH domain with selected mammalian PH domains. Secondary structure elements (green arrows, $\beta$-strands; blue cylinder, $\alpha$-helix) depict those in the crystal structure of the PLC $\delta 1 \mathrm{PH}$ domain (Lemmon and Keleti 2005). Basic residues in the $\beta 1-\beta 2$ loop and the conserved Trp in the C-terminal helix are highlighted (bold). (C) Secondary structure elements in the Ste5 $\mathrm{PH}$ domain predicted by the indicated algorithms: 3D-PSSM, SSPro, YAPSIN, PSIPRED, and SYMPRED. (Green/E) $\beta$-strand; (blue/H) $\alpha$-helix; (C) random coil.

protein, with or without the $\mathrm{PH}$ domain mutations. As a positive control, a GST fusion to the PH domain of mammalian phospholipase C $\delta 1$ (PLC $\delta 1)$, which binds well to $\operatorname{Ptd} \operatorname{Ins}(4,5) \mathrm{P}_{2}$, was expressed and purified in a similar manner. Using an overlay assay in which the GST-PH domain fusions were incubated with various lipids immobilized on nitrocellulose membranes (Yu et al. 2004), we found that the Ste5 PH domain bound to $\operatorname{PtdIns}(4,5) \mathrm{P}_{2}$ and did so, in most experiments, at least as specifically as the PLC $\delta 1$ PH domain (Fig. 3A). Most importantly, no detectable binding of the $\mathrm{PH}$ domain of the mating-defective mutant, Ste5(R407S K411S), was ever observed by this method (Fig. 3A), whereas the nondefective Ste5(K416S K420S) mutant still displayed detectable binding (data not shown).

To confirm the reliability of these results, binding of the same Ste5 PH domain constructs to phospholipid vesicles in solution was also examined. In this assay, artificial PtdCho/PtdEth liposomes without or with an increasing mole fraction of different phosphoinositides (and marked with Texas Red to follow the efficiency of vesicle recovery) were incubated with the GST-PH domain fusions and separated from excess unbound protein by flotation through a sucrose gradient. In agreement with the overlay assay, the Ste5 PH domain bound best to liposomes containing PtdIns $(4,5) \mathrm{P}_{2}$, and did so distinctly more avidly than the Ste5(R407S K411S) mutant (Fig. 3B). Indeed, in multiple trials, the R407S K411S mutations decreased PtdIns $(4,5) \mathrm{P}_{2}$ binding of the Ste5 $\mathrm{PH}$ domain to the background level (Fig. 3C). Based on the percent recovery at equivalent input concentrations, the Ste5 PH domain bound significantly more weakly than did the PLC $\delta 1$ PH domain (data not shown). However, as expected for authentic binding, the amount of vesicle- 


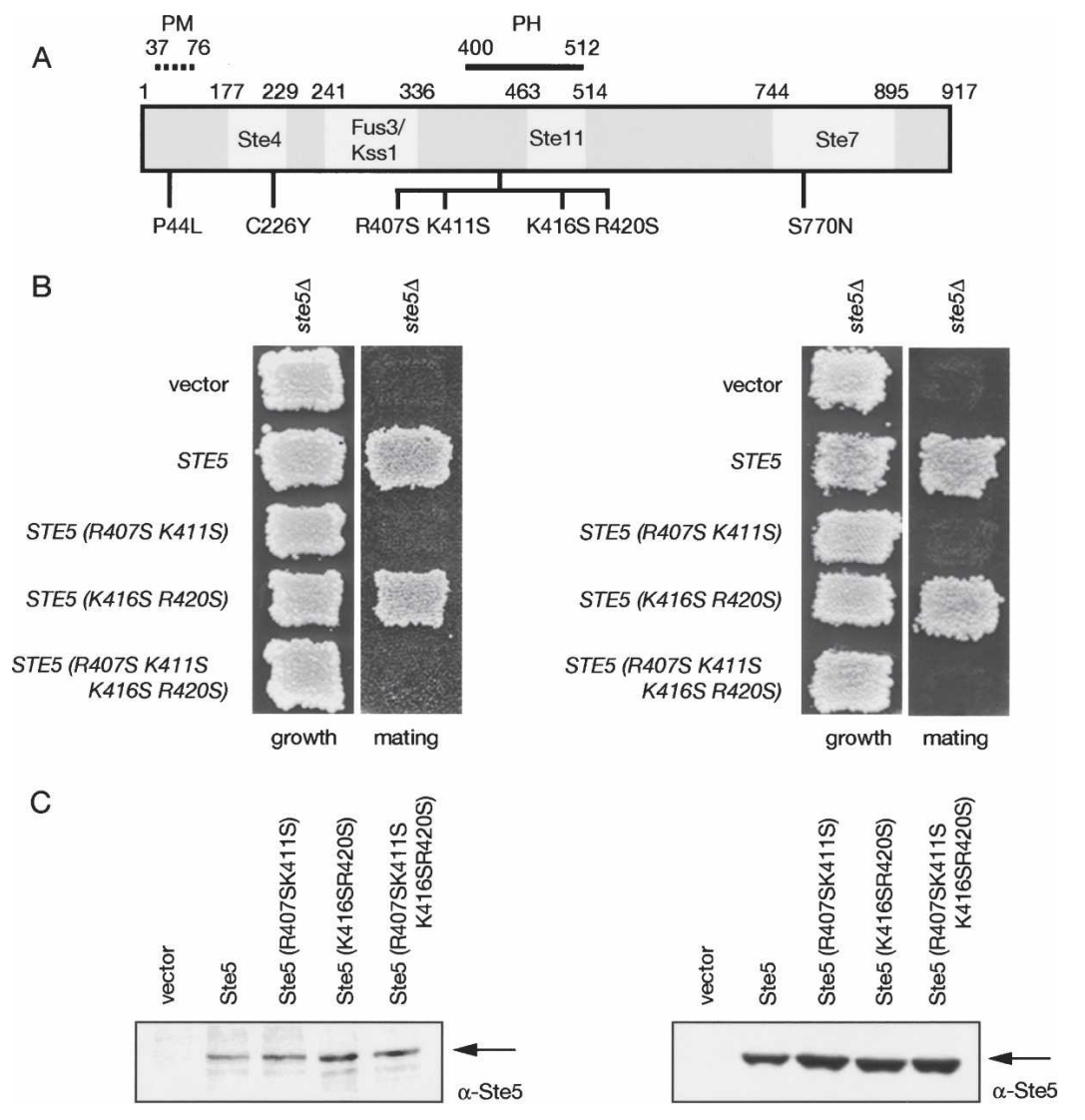

Figure 2. The PH domain is essential for Ste5 function in vivo. (A) Ste5 primary structure. (White boxes) Regions implicated in binding the indicated proteins; (dotted line) location of the PM motif; (solid line) location of the predicted $\mathrm{PH}$ domain. Positions of previously characterized hyperactive alleles (P44L, C226Y, S770N) and basic residues mutated in this study (R407S K411S R416S R420S) are indicated. (B) Strain BYB69 (ste5 5 ) was transformed with either an empty $C E N$ vector or the same vector expressing wildtype Ste5, Ste5(R407S K411S), Ste5(K416S R420S), or Ste5(R407S K411S K416S R420S) from either the native STE5 promoter (left) or from the GAL1 promoter (right), and patch-mating assays were carried out as described in Materials and Methods. $(C)$ Extracts of the same cultures as in $B$ were prepared, resolved by SDS-PAGE, and analyzed by immunoblotting with either an $\alpha$-Ste 5 antiserum (gift of K. Benjamin) (left) or affinity-purified $\alpha$-Ste5 IgG (Hasson et al. 1994) (right). bound GST-Ste5 PH domain (but not GST alone) increased commensurately with an increase in the PtdIns $(4,5) \mathrm{P}_{2}$ content of the liposomes (Fig. 3D) or with an increase in the amount of the GST-Ste5 $\mathrm{PH}$ protein added (data not shown).

The same trends were observed using a third method, surface plasmon resonance, in which the $\mathrm{PH}$ domain constructs were flowed over vesicles covalently immobilized (via phytosphingosine) to the derivatized goldplated surface of the detector cell: The Ste5 $\mathrm{PH}$ domain bound to PtdIns $(4,5) \mathrm{P}_{2}$-containing liposomes, and bound markedly better than the R407S K411S mutant over the range of protein concentrations tested (data not shown). We conclude that, like certain other isolated S. cerevisiae $\mathrm{PH}$ domains examined previously (Yu et al. 2004), the Ste5 $\mathrm{PH}$ domain binds PtdIns $(4,5) \mathrm{P}_{2}$ directly and reasonably specifically, but with relatively modest affinity. Nonetheless, taken together with the mating results, these findings suggested that the $\mathrm{PH}$ domain could have a direct and critical role in membrane recruitment of Ste5 and that the mating-defective behavior of the R407S K411S mutant might be explained by its inability to be targeted to and stably associate with the plasma membrane.

\section{The PH domain is required for membrane association} of Ste5

To examine membrane recruitment in live cells in real time, Ste5-GFP fusions with or without the $\mathrm{PH}$ domain mutations were constructed and expressed as the sole source of Ste5 in MATa haploids. After exposure to pheromone ( $\alpha$-factor), cells expressing either wild-type Ste5 or the functional Ste5(K416S R420S) mutant formed the typical "shmoo" shape, and a significant fraction of both proteins was rapidly relocalized to the cortex at the elongating cell pole (Fig. 4A, left and right), as observed for Ste5 by us and others previously (Pryciak and Huntress 1998; Mahanty et al. 1999; Sette et al. 2000). Ste5 and Ste5(K416S R420S) remained stably associated with the shmoo tip throughout the time period examined (up to $1 \mathrm{~h})$. In contrast, cells expressing the nonfunctional Ste5(R407S K411S) mutant did not form shmoos and the protein was not recruited to the plasma membrane, even though the cells contain G $\beta \gamma$, previously thought to be the major recruiter of Ste5 to the cell cortex (Fig. 4A, middle). We noted that Ste5(R407S K411S) also displayed elevated cytoplasmic fluorescence compared with wildtype Ste5; this increase was not due to any defect in nuclear import because Ste5(R407S K411S) accumulated in the nucleus to the same extent as wild-type Ste5 in an msn5s mutant in which pheromone-induced nuclear export is blocked (L.S. Garrenton, unpubl.). Unlike Ste5(R407S K411S), other Ste5 derivatives that exhibit an increased cytoplasmic level (e.g., Ste5-GST fusions) show an increase in membrane recruitment and constitutive activation of the mating pathway (Inouye et al. 1997b; Wang and Elion 2003).

Given that Ste5(R407S K411S)-GFP is signaling-defec- 
A

Figure 3. The Ste5 PH domain binds $\operatorname{PtdIns}(4,5) \mathrm{P}_{2}$. (A) Overlay on immobilized lipids. The indicated purified GST fusions (1 $\mu M)$ were incubated with commercial filter strips (three right-most panels) on which the indicated phospholipids and related compounds were spotted in the pattern shown (left). (B) Binding to liposomes. Samples (1 $\mu \mathrm{M}$ ) of the indicated purified GST fusions (input) were incubated with synthetic vesicles of the following compositions: PC/PE $177 \%$ DOPC, $22 \%$ DOPE); PI (52\% DOPC, $22 \%$ DOPE, 10\% DOPS, 5\% DOPA, 10\% PI); $\mathrm{PI}(4) \mathrm{P}$; and PIP2 [same as PI vesicles, except $5 \% \mathrm{PI}$ and $5 \% \mathrm{PI}(4) \mathrm{P}$ or $5 \% \mathrm{PI}(4,5) \mathrm{P}_{2}$, respectively], each marked with $1 \%$ Texas Red-PE. $(C)$ Summary of liposome-binding assays. Values, obtained as in $B$, represent the amount of vesicle-bound protein (expressed as percent of input) averaged over three to four independent trials. Bars indicate standard error. $(D)$ Effect of vesicle content of $\operatorname{PtdIns}(4,5) \mathrm{P}_{2}$. Binding assays were performed with GSTSte5 PH domain or GST alone, as a control, as in $B$ with liposomes containing the indicated fraction of $\mathrm{PI}(4,5) \mathrm{P}_{2}$ (when PIP2 exceeded $10 \%$, DOPC was decreased correspondingly).

B

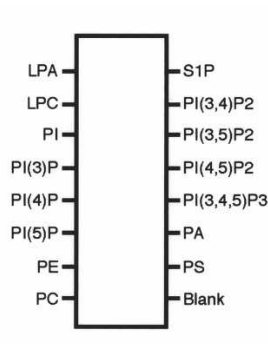

GST-Plc $\delta$-1 PH GST-Ste5 PH GST-Ste5 (R407S K411S) PH
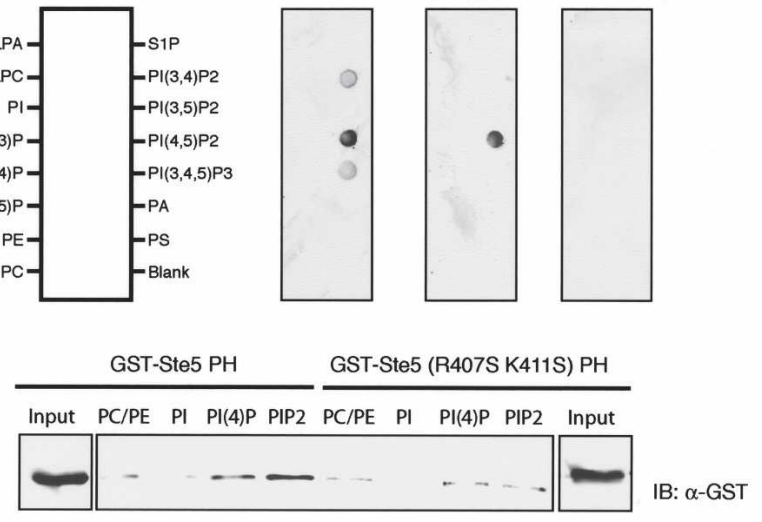

C

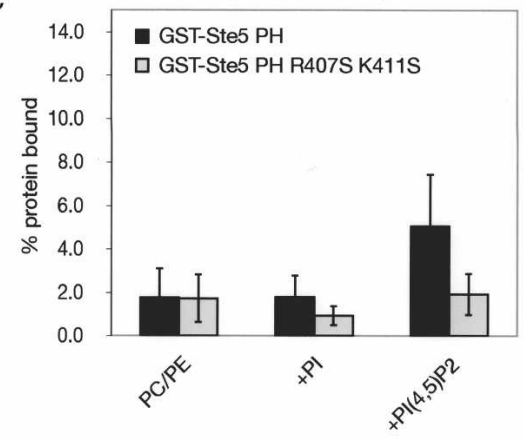

D
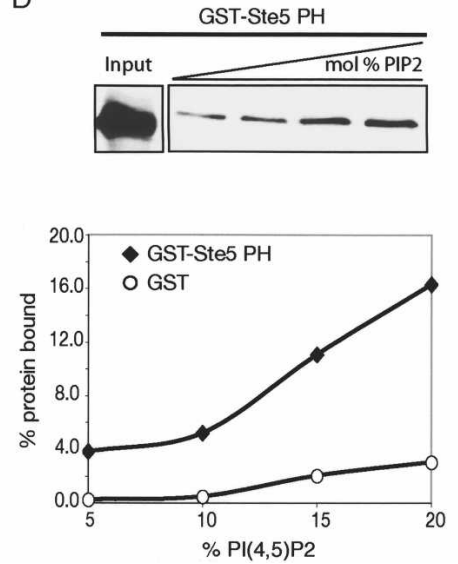

tive, its failure to be recruited to the plasma membrane at the shmoo tip might arise simply because the cells do not undergo pheromone-induced morphogenesis. To rule out this possibility, we stimulated G $\beta \gamma$ release in cells (ste11D) in which downstream MAPK signaling was blocked, thereby preventing subsequent shmoo formation (for review, see Knaus et al. 2005). Under these conditions, wild-type Ste5 and Ste5(K416S R420S) were recruited to numerous disperse membrane-associated puncta (Fig. 4B, left and right), whereas Ste5(R407S K411S) remained in the cytosol (Fig. 4B, middle). Thus, under all conditions tested, and in agreement with its inability to bind to phosphoinositide-containing vesicles in vitro, Ste5(R407S K411S) was completely defective in membrane recruitment in response to pheromone, indicating that the $\mathrm{PH}$ domain plays an essential role in Ste5 association with the plasma membrane in vivo. Moreover, this role of the $\mathrm{PH}$ domain lies upstream of the MAPK cascade and is required even when G $\beta \gamma$ is present.

Membrane targeting rescues the mating defect of the Ste5 PH domain mutant

If the sole role of the $\mathrm{PH}$ domain in Ste5 function is to help anchor this scaffold protein to the plasma membrane, then the signaling defect of the Ste5(R407S K411S) mutant should be overcome completely by tethering the protein to the plasma membrane by other means. To test this prediction, two different membrane targeting elements-the C-terminal transmembrane segment (CTM) of yeast Snc2 (a synaptobrevin-like R-type v-SNARE) and the S-palmitoylated and S-farnesylated C-terminal CCAAX box of yeast Ras2-were fused in-frame to the C terminus of Ste5 lacking or containing the $\mathrm{PH}$ domain mutations (Fig. 5A). As an additional control, we also used a C318S C319S derivative of the Ras2 CCAAX box that cannot be modified by any lipophilic substituent and consequently cannot mediate membrane association (Mitchell and Deschenes 1995). Prior work has shown that fusion of the Snc2 CTM and the Ras2 CCAAX box to wild-type Ste5 does not perturb its function, but rather bypasses the need for G $\beta \gamma$ and constitutively activates downstream signaling (Pryciak and Huntress 1998). Indeed, we found that attachment of the CTM and the CCAAX box permitted both normal Ste5 and the functional Ste5(K416S R20S) mutant to fully rescue the mating defect of ste $5 \Delta$ cells (Fig. 5B, top and bottom). Consistent with the conclusion that the sole defect conferred by the nonfunctional PH domain mutant is a lack of membrane association, attachment of the CTM and the CCAAX box (but not the mutant SSAAX box) also fully restored mating in ste5s cells expressing Ste5(R407S K411S) (Fig. 5B, middle). Furthermore, as as- 
A
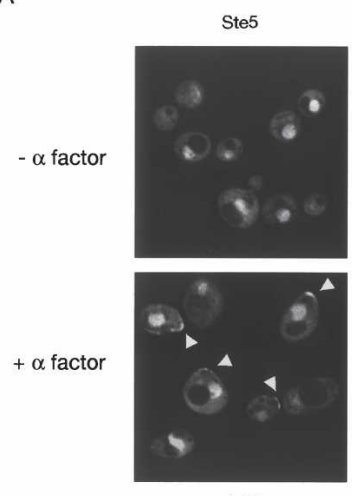

ste5s

B

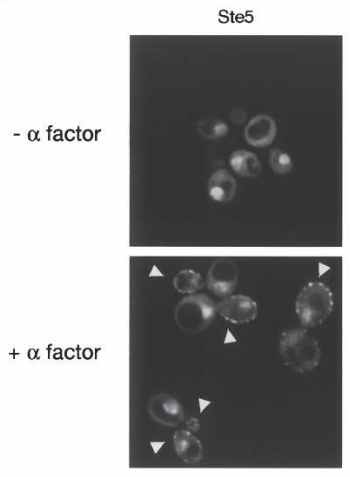

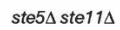

Ste5 R407S K411S
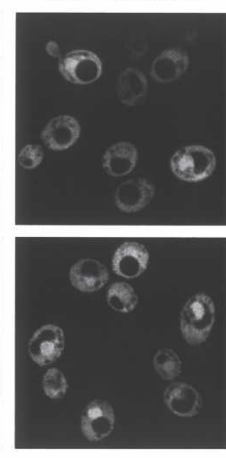

ste5s

Ste5 R407S K411S
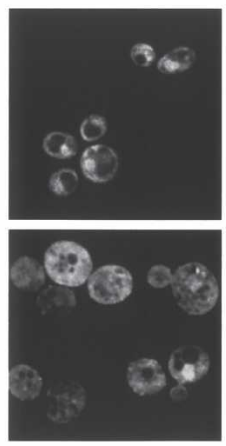

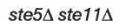

Ste5 K416S R420S
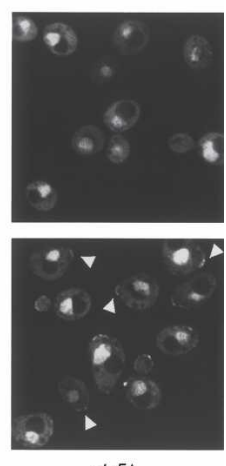

ste5 $\Delta$

Ste5 K416S R420S
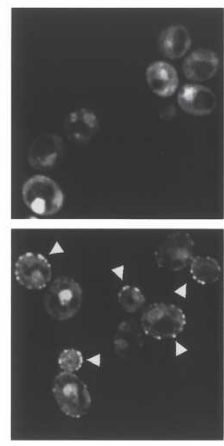

ste5 5 ste11 14

Figure 4. The $\mathrm{PH}$ domain is required for pheromone-induced membrane recruitment of Ste5. Strain BYB84 (ste5s) $(A)$ or strain YAS1 (ste5 ste11 $)(B)$ transformed with CEN plasmids expressing from the GAL1 promoter wild-type Ste5-GFP (pCJ80), Ste5(R407S K411S)-GFP (pLG35), or Ste5(K416S R420S)-GFP (pLG36) were grown, induced with galactose, treated with pheromone for $1 \mathrm{~h}$, and viewed by deconvolution microscopy, as indicated in Materials and Methods. Panels depict a collage of representative images; arrowheads indicate Ste5-GFP accumulated at the cell cortex.

sessed by the level of expression of a pheromone-responsive reporter gene, FUS1::HIS3, the Ste5(R407S K411S) mutant is fully capable of propagating a signal from a dominant allele (STE11-4), which produces a constitutively active kinase, Ste11(T596I), that bypasses the need for its activation at the membrane by Ste20 (Supplementary Fig. S1; Stevenson et al. 1992). Thus, the Ste5 PH domain mutations prevent Ste5 from functioning only when signaling is initiated at the plasma membrane, but not when the need for membrane recruitment is bypassed by artificial targeting or by an activated allele of Ste11. Together, these data argue strongly that the only defect of Ste5(R407S K411S) is its demonstrated inability to be recruited to the plasma membrane and, therefore, that the $\mathrm{PH}$ domain is essential for Ste5 interaction with the membrane.

\section{Ste5 PH domain mutant binds MAPKKK Ste11}

The predicted $\mathrm{PH}$ domain (400-512) partially overlaps with the region (residues 463-514) previously implicated

in binding of Ste11 to Ste5 (Fig. 2A). Indeed, it was concluded that Ste11 binding is required for Ste 5 membrane recruitment because mutations in this region [e.g., Ste5(4474-487)] prevented its association with both Ste11 and the plasma membrane (Wang and Elion 2003). However, the altered region corresponds to the C-terminal portion of the $\mathrm{PH}$ domain. In contrast to alterations of the C-terminal end of the $\mathrm{PH}$ domain, the nonfunctional Ste5(R407S K411S) and Ste5(R407S K411S K416S R420S) mutants associate with Ste11 indistinguishably from wild-type Ste5 or the functional Ste5(K416S R420S) mutant, as judged by coimmunoprecipitation from cell extracts, regardless of whether Ste11 was overexpressed (Fig. 6A, left) or expressed at its endogenous level from its chromosomal locus (Fig. 6A, right). Thus, the R407S K411S mutations that abrogate the function of the Ste5 PH domain do not alter the affinity of Ste5 for Ste11.

\section{Ste5 PH domain mutant oligomerizes}

Prior work indicates that Ste5 self-associates and that this oligomerization is important for its signaling function (Yablonski et al. 1996; Inouye et al. 1997b; Sette et al. 2000; Wang and Elion 2003). The PH domain lies within one of two regions in Ste5 (residues 335-586) that have been implicated in mediating its oligomerization. To determine whether the signaling defect of Ste5(R407S K411S) could be explained by an inability to self-associate, three independent and complementary approaches

A

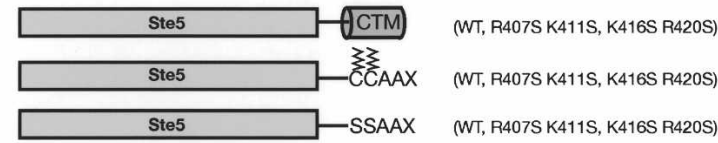

\begin{tabular}{|lcrc|} 
Name & Source & Sequence & Localization \\
\hline CTM & Snc2 & --WWKDLKMRMCLFLVVILLVVIIVPIVVHFS & PM \\
CCAAX & Ras2 & --APGGNTSEASKSGSGGCCIIS & PM \\
SSAAX & ras2-SSIIS & --APGGNTSEASKSGSGGsSIIS & cytoplasm \\
\hline
\end{tabular}

B

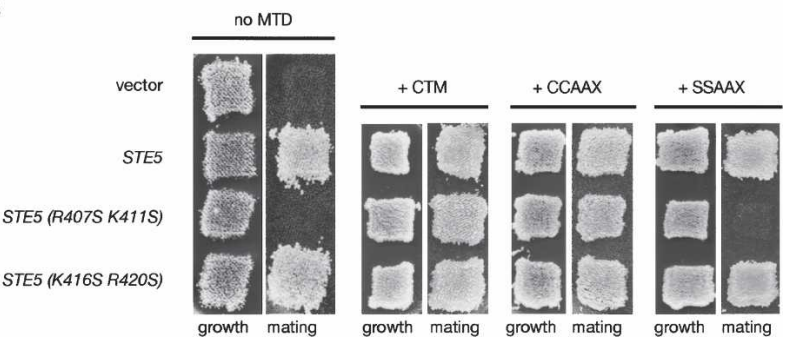

Figure 5. Forced membrane anchoring rescues the Ste5 $\mathrm{PH}$ domain mutant. (A) Membrane targeting elements. CTM (cylinder), C-terminal $\alpha$-helical transmembrane segment of Snc2; Spalmitoylated and S-farnesylated (zig-zags) C-terminal segment of wild-type (CCAAX) and unmodified mutant (SSAAX) Ras2. (B) Mating proficiency of BYB69 (ste5s) cells expressing either empty vector, wild-type Ste5, or the indicated Ste5 mutants, not fused to any membrane targeting domain (no MTD) or fused to the elements shown in $A$, was analyzed using a patch-mating assay as in Figure 2B. 
Figure 6. The Ste5 $\mathrm{PH}$ domain mutant retains MAPKKK binding and capacity to oligomerize. (A) Ste11 binding. Strain YAS1 (ste5s ste11 $)$ (left)-expressing from the GAL1 promoter either a vector control (-) or c-Myc epitope-tagged versions of wild-type Ste5 or the indicated Ste5 $\mathrm{PH}$ domain mutants, and coexpressing from the GAL1 promoter either a vector control (-) or Ste11 (+)-was lysed, and the resulting extracts were subjected to immunoprecipitation using $\alpha$-c-Myc monoclonal antibody, 9E10, as indicated in Materials and Methods. The resulting immune complexes were resolved by SDS-PAGE and analyzed by immunoblotting with either affinity-purified $\alpha$-Ste5 IgG (left) or another $\alpha$-Ste5 antiserum (gift of K. Benjamin) (right), or with $\alpha$-Ste11 antisera, as indicated. Samples $(80 \mu \mathrm{g})$ of the whole-cell extracts (WCE) were analyzed by immunoblotting to confirm equivalent levels of expression of the expected proteins. Strain YLG14 (ste5s STE11::3xHA) (right)—not expressing or expressing the same Ste5 constructs as on the left, and expressing an HA epitope-tagged version of Ste11 from its native promoter at its endogenous chromosomal lo-

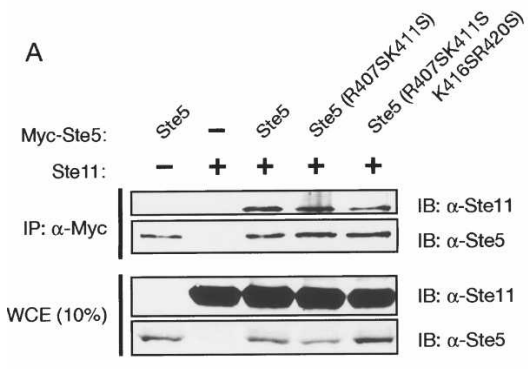

B

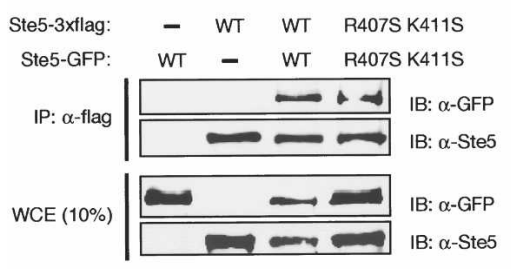

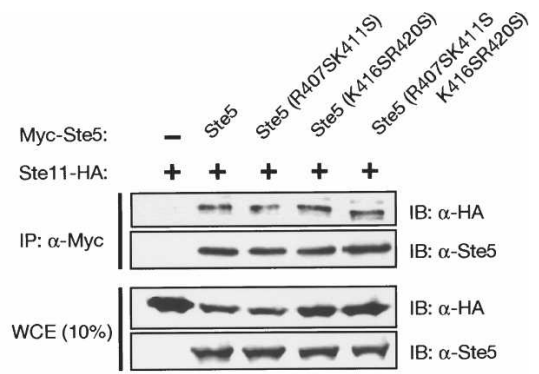

C

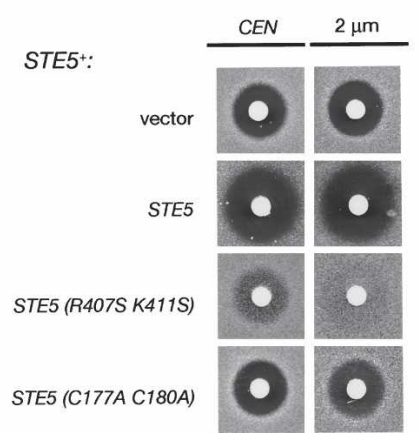

cus-was lysed, and the resulting extracts were subjected to immunoprecipitation and analysis as on the left, except mouse monoclonal $\alpha$-HA antibodies were used for detection of Ste11-HA . $_{3}$ (B) Ste5 self-association. Strain YLG19 (ste $4 \Delta$ ste $\left.5 \Delta\right)$-expressing from the GAL1 promoter either vector controls (-) or coexpressing 3xFlag epitope- and GFP-tagged versions of either wild-type Ste5 or Ste5(R407S K411S), as shown-was lysed, and the resulting extracts were subjected to immunoprecipitation using anti-Flag M2 affinity gel (Sigma), as indicated in Materials and Methods. The resulting immune complexes and cell extracts (WCE) were resolved by SDS-PAGE and analyzed by immunoblotting with either mouse monoclonal $\alpha$-GFP antibodies or affinity-purified $\alpha$-Ste5 IgG. (C) Potent dominant-negative effect of Ste5(R407S K411S). Standard halo bioassays of pheromone response (15 $\mu$ g $\alpha$-factor spotted on each filter disk) were performed using strain BY4741(STE5 ${ }^{+}$expressing either empty vector, wild-type Ste5, or the indicated Ste5 mutants from the GAL1 promoter on either a CEN (left) or 2-um (right) vector.

were taken. First, we coexpressed in MATa ste $4 \Delta$ ste $5 \Delta$ cells two differentially tagged versions of Ste5(R407S K411S), one fused to GFP and the other containing a 3xFlag epitope. Both derivatives of Ste5(R407S K411S) were coimmunoprecipitated (using anti-Flag antibodycoated beads) just as efficiently as the same derivatives of wild-type Ste5 (Fig. 6B). Second, when expressed in wildtype cells, Ste5(R407S K411S) acts as a potent dominantnegative (Fig. 6C). By contrast, overexpression of an oligomerization-defective mutant, Ste5(C177A C180A), has a significantly weaker effect. These results indicate that the observed inhibition by the Ste5 PH domain mutant cannot be due merely to titration of signaling components, but rather to formation of mixed oligomers with reduced capacity for membrane binding. Third, the mating defect of the Ste5(R407S K411S) mutant was not rescued by forced dimerization with a heterologous dimerization domain, GST (Maru et al. 1996), which as we previously have shown restores signaling function to known oligomerization-defective mutants of Ste5 (Supplementary Fig. S2). Hence, the inability of Ste5(R407S K411S) to function in vivo reflects a role for the $\mathrm{PH}$ domain in a property of Ste5 different from oligomerization, consistent with the other evidence presented for its essential role in membrane recruitment of Ste5.
The PH domain is not required for G $\mathrm{\beta} \gamma$ binding to Ste5

Interaction of Ste 5 with membrane-anchored G $\beta \gamma$ freed upon exposure of cells to pheromone is an early, crucial, and well-established event in this signaling pathway (for review, see Wang and Dohlman 2004). Given the inability of Ste5(R407S K411S) to be recruited to the membrane in response to pheromone, it was possible that this $\mathrm{PH}$ domain mutant was unable to interact with $\mathrm{G} \beta \gamma$, in addition to its defect in binding to membrane phosphoinositides revealed by our in vitro experiments. However, as judged by coimmunoprecipitation from cell extracts, the nonfunctional mutants, Ste5(R407S K411S) and Ste5(R407S K411S K416S R420S), showed no detectable diminution in their ability to associate with Ste4 (Gß), compared with wild-type Ste5 or the functional mutant, Ste5(K416S R420S), expressed under the same conditions (Fig. 7A).

Elevated expression of Ste5 leads to pathway activation, resulting in hyperphosphorylation of Ste4 (Cole and Reed 1991). Indeed, hyperphosphorylated Ste4 was the primary species present in extracts of cells expressing wild-type Ste5 or the functional Ste5(K416S R420S) mutant and coimmunoprecipitated with both of these proteins, whereas hypophosphorylated Ste4 was the major 
A

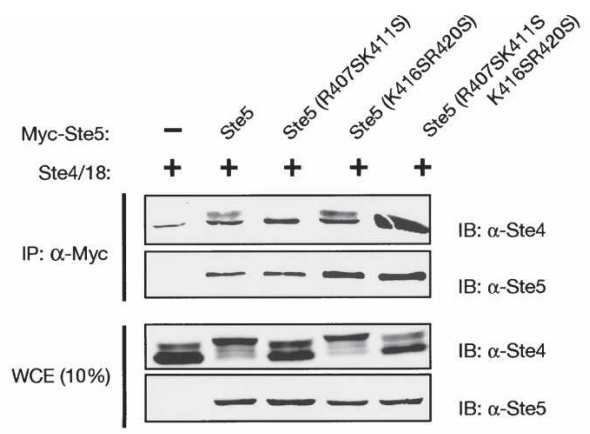

Figure 7. The Ste5 PH domain acts separately from, but synergistically with, G $\beta \gamma$. $(A)$ The Ste5 PH domain mutant retains G $\beta \gamma$ binding. Strain BYB84 (ste $5 \Delta$ )-expressing from the GAL1 promoter either a vector control (-) or the c-Myc epitope-tagged Ste5 derivatives described in Figure 5A and coexpressing STE4 and STE18 under the control of the divergent GAL1-GAL10-was lysed, subjected to immunoprecipitation, and analyzed by immunoblotting as in Figure 6A using $\alpha$-Ste 5 antisera and $\alpha$-Ste 4 antisera. A trace of Ste 4 associates with antibody-coated beads nonspecifically in the absence of Ste5, as we have observed previously (Inouye et al. 1997a). (B) Loss of $\mathrm{PH}$ domain function abrogates the constitutive activity of hyperactive Ste5 alleles. Strains

B STE5 (P44L) STE5 (S77ON) STE5 (C226Y)
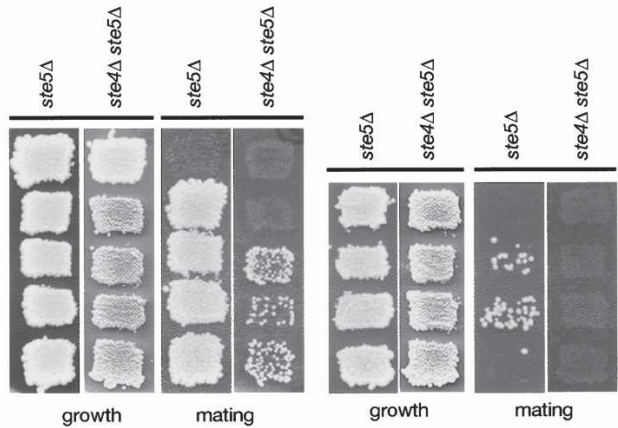

STE5 (R407S K411S) STE5 (P44L R407S K411S) STE5 (S770N R407S K411S) STE5 (C226Y R407S K411S)

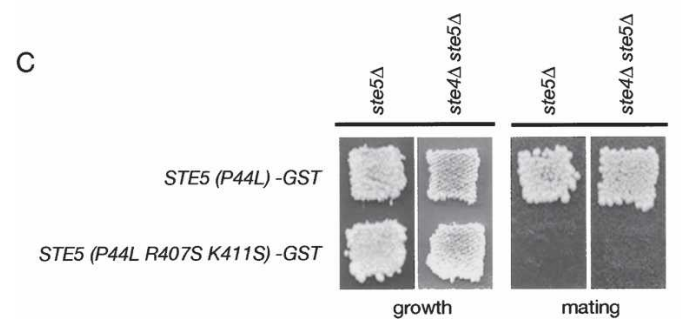
BYB69 (ste $5 \Delta$ ) or BYB88 (ste $4 \Delta$ ste $5 \Delta$ ) were transformed with empty $2 \mu \mathrm{m}$-DNA vector or the same vector expressing from the GAL1 promoter either wild-type Ste5 or Ste5(R407S K411S), Ste5(P44L) or Ste5(P44L R407S K411S), Ste5(S770N) or Ste5(R407S K411S S770N), or Ste5(C226Y) or Ste5(C226Y R407S $\mathrm{K} 411 \mathrm{~S}$ ), as indicated, and patch-mating assays were done as in Figure 2B. $(C)$ Loss of PH domain function still abrogates the enhanced activity of the PM motif mutant, Ste5(P44L)-GST. Strain BYB88 (ste4A ste $5 \Delta$ ) was transformed with a CEN vector expressing from the GAL1 promoter either Ste5(P44L)-GST or Ste5(P44L R407S K411S)-GST, and patch-mating assays were performed. species present in extracts of cells expressing the nonfunctional Ste5(R407S K411S) or Ste5(R407S K411S K416S R420S) mutants and coimmunoprecipitated with those proteins (Fig. 7A). Thus, as judged by this additional indicator of early events in the response pathway, the $\mathrm{PH}$ domain mutations render Ste5 incompetent to transmit a pheromone-induced signal, and this defect is not due to an inability to associate with G $\beta \gamma$. The above observations, taken together with the results of our analysis of subcellular localization (Fig. 4), make it clear that, in the absence of a functional PH domain, interaction with $G \beta \gamma$ is not sufficient for membrane recruitment of Ste5 in response to pheromone.

\section{Hyperactive Ste5 alleles do not function in the absence of an intact PH domain}

Our initial impetus for examining mechanisms for membrane targeting of Ste 5 other than by its interaction with G $\beta \gamma$ was the fact that we were able to generate hyperactive Ste5 alleles that promote signaling even when G $\beta \gamma$ is absent (Hasson et al. 1994; Sette et al. 2000). We reasoned that the activating mutations must enhance the ability of Ste5 to associate with the membrane. Indeed, the apparent explanation for two such alleles (P44L and
T52M) is that they install markedly more hydrophobic residues into an $\mathrm{N}$-terminal basic $\alpha$-helix (PM motif), thereby elevating its intrinsic propensity to associate with acidic phospholipids (Winters et al. 2005). We reasoned further, however, that such an interaction may not be sufficient and that effective membrane recruitment of Ste5, even by these hyperactive alleles, may depend on synergy between binding of the $\mathrm{PH}$ domain to phosphoinositides and binding of the mutant PM motif to the membrane.

To test this possibility, we introduced the $\mathrm{PH}$ domain loss-of-function mutations, R407S K411S, into Ste5 mutants containing each of three different gain-of-function mutations: P44L, C226Y, and S770N. As observed before, wild-type Ste5 and all three hyperactive alleles (P44L, C226Y, and S770N) promoted robust mating in ste5 $\Delta$ cells, whereas only the hyperactive alleles supported detectable mating in a ste $4 \Delta$ ste $5 \Delta$ double mutant (Fig. 7B, left). However, when the same hyperactive mutants were combined with the R407S K411S PH domain mutations, no mating occurred in the cells lacking G $\beta \gamma$ (ste $4 \Delta$ ste $5 \Delta$ strain) (Fig. 7B, right). Moreover, introduction of the R407S K411S mutations completely blocked signaling even when Ste5(P44L) was fused to GST, which greatly enhances the potency of the $\mathrm{P} 44 \mathrm{~L}$ allele in pro- 
moting mating (Fig. 7C), in agreement with prior work (Sette et al. 2000; Winters et al. 2005). Interestingly, in cells containing G $\beta \gamma$ (ste $5 \Delta$ strain), two of the hyperactive alleles supported a detectable degree of mating, even when the $\mathrm{PH}$ domain was ablated (Fig. 7B, right).

These observations indicate, first, that the hyperactive mutants cannot bypass the need for G $\beta \gamma$ when the PH domain is not functional. Second, the fact that the $\mathrm{PH}$ domain is essential for the hyperactive alleles to function in cells (ste $4 \Delta$ ste $5 \Delta$ ) that completely lack G $\beta \gamma$ confirms that the $\mathrm{PH}$ domain contributes to membrane recruitment in a manner that does not depend on its direct interaction with G $\beta \gamma$. Third, the fact that the presence of $\mathrm{G} \beta \gamma$ partially suppressed the mating debility of the $\mathrm{PH}$ domain-defective hyperactive alleles provides further support for the conclusion that the $\mathrm{PH}$ domain works independently from, but synergistically with, G $\beta \gamma$ in recruitment of Ste5 to the membrane. Collectively, these findings show that no single mode of membrane interaction (via the $\mathrm{PH}$ domain, the mutant PM motif, or G $\beta \gamma$ binding) is sufficient for signaling, whereas inefficient signaling can be mounted by any combination of two of these modes, and that maximal signaling requires all three (Fig. 8).

\section{Discussion}

Our results demonstrate that a previously uncharacterized PH domain in Ste5 has an essential role in the function of this archetypal MAPK scaffold protein. Alteration of two basic residues in this domain abogrates the ability of Ste 5 to promote signaling in response to pheromone, prevents Ste5 binding to a specific phosphoinositide in vitro, and eliminates membrane recruitment of Ste 5 in vivo. These mutations do not affect any of the other functions of Ste5 attributed to this region of the protein (such as binding of Ste11 or oligomerization) or G $\beta \gamma$ binding. Thus, interaction of Ste 5 with G $\beta \gamma$ is not sufficient for membrane tethering when the $\mathrm{PH}$ domain is nonfunctional. Fusion of the Ste5 $\mathrm{PH}$ domain mutant to the CTM domain of Snc2 or the CCAAX box of Ras2 efficiently ameliorates its signaling defect; thus, alternative means of membrane binding bypass the need for the $\mathrm{PH}$ domain. Even when the membrane-associating propensity of Ste 5 is elevated by means intrinsic to Ste5 (such as mutations, like P44L, that increase the membrane affinity of the N-terminal PM motif) and G $\beta \gamma$ is available, signaling is still very inefficient when the $\mathrm{PH}$ domain is not functional. Conversely, the $\mathrm{PH}$ domain is not necessary to propagate a signal initiated by Ste11(T596I), a constitutively active mutant of the MAPKKK that bypasses the need for Ste20-initiated signaling at the plasma membrane. Hence, it seems clear that optimal function of Ste5 normally requires stable membrane recruitment via concerted action of the $\mathrm{PH}$ domain, the PM motif, and binding to G $\beta \gamma$ (Fig. 8).

At present, based on estimates from our in vitro phospholipid-binding studies (L.S. Garrenton, unpubl.), interaction of the Ste5 $\mathrm{PH}$ domain with $\operatorname{PtdIns}(4,5) \mathrm{P}_{2}$ has a $\mathrm{K}_{\mathrm{d}}=\sim 5-10 \mu \mathrm{M}$, which is significantly weaker than that of the well characterized PtdIns $(4,5) \mathrm{P}_{2}$-specific PLC 81 $\mathrm{PH}$ domain $\left(\mathrm{K}_{\mathrm{d}}=200 \mathrm{nM}\right)$ (Lemmon and Keleti 2005). However, this property is not uncommon among PH domains. Only a small minority of known $\mathrm{PH}$ domains binds their cognate phosphoinositide with high affinity and specificity, whereas the large majority binds phosphoinositides less selectively and rather weakly $\left(\mathrm{K}_{\mathrm{d}}=10-20 \mu \mathrm{M}\right.$ or greater) (Yu et al. 2004). However, the specific nature of the Ste5 PH domain may be physiologically important because it may set a threshold that prevents inadvertent association of Ste 5 with the membrane in the absence of authentic pheromone stimulation. Moreover, it may explain, at least in part, the apparently vital role of oligomerization in Ste5 function (Yablonski et al. 1996; Inouye et al. 1997b), given that multimerization of $\mathrm{PH}$ domains and other membranebinding motifs greatly increases their membrane-binding avidity (Stefan et al. 2002; Winters et al. 2005). For example, the $\mathrm{PH}$ domain of mammalian dynamin-1 requires oligomerization for significant membrane association, and, when overexpressed in vivo, $\mathrm{PH}$ domain-defective dynamin-1 mutants act as dominant-negative inhibitors (Lemmon and Keleti 2005), as we have observed for Ste5(R407S K411S). In addition, the relatively modest affinity of the $\mathrm{PH}$ domain for membrane phosphoinositide may explain why it must act in cooperation with G $\beta \gamma$ binding and the PM motif to achieve effective membrane association. Multiple, but low-affinity, membrane targeting domains make pathway activation dependent on coincident and highly cooperative detection of both receptor occupancy and a receptive membrane
Figure 8. Requirement for the $\mathrm{PH}$ domain in membrane docking and signaling by Ste5. Coordinate action of G $\beta \gamma$, the PM motif, and the PH domain is required for stable recruitment of Ste5 at the membrane and robust pathway activation. For clarity, other pathway components have been omitted. Adapted from Winters et al. 2005 with permission from Elsevier (c) 2005.
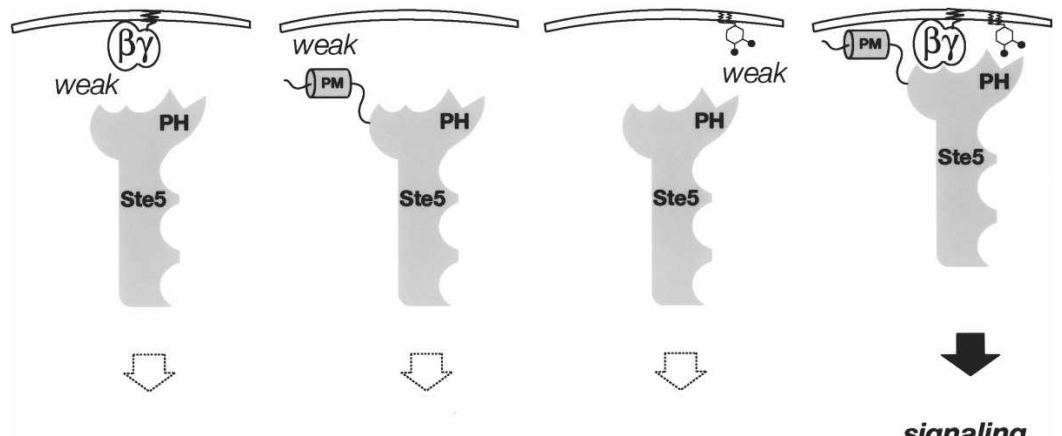

signaling 
milieu. Multiple sites of interaction, once joined, also provide relatively stable membrane localization, imposing spatial selectivity and thus a mechanism to ensure that only Ste5-bound Ste11 becomes activated.

Coordinate action of a $\mathrm{PH}$ domain with other membrane interaction and/or protein interaction motifs to drive membrane association is not unique to Ste5. The $\mathrm{PH}$ domain of the $\beta$-adrenergic receptor kinase, Grk2, binds both phosphoinositides and G $\beta \gamma$, yet neither interaction alone supports efficient membrane recruitment (Lemmon and Keleti 2005). Likewise, the PH domain in a yeast PAK, Cla4 (Wild et al. 2004), and PH domains in PLC $\gamma 1$ and in a Rho- and Rac GEF, Tiam1 (Lemmon and Keleti 2005), all act in conjunction with additional domains to achieve proper membrane recruitment and localization. Most tellingly, in S. cerevisiae pheromone response, another scaffold protein, Farl, serves to deliver the Cdc42 GEF, Cdc24, to the plasma membrane. Sequence alignments show that Farl shares two regions of strong similarity with Ste5: an N-terminal RING-H2 domain (residues 202-251), which interacts with G $\beta \gamma$ (Butty et al. 1998), just like its counterpart in Ste5 (Inouye et al. 1997b; Feng et al. 1998); and a more internal segment (residues 416-550), corresponding to the region in Ste5 that we have demonstrated here contains its functional PH domain. Despite the presence of its G $\beta \gamma$ binding RING-H2 domain, deletion of the PH domainlike element in Farl prevents its recruitment to the shmoo tip during pheromone response (Wiget et al. 2004).

In this same regard, prior work has suggested that interaction of Ste 5 with other proteins, specifically Bem 1 and Cdc24, contributes to recruiting Ste5 to the shmoo tip to promote pheromone-induced signaling (Leeuw et al. 1995; Lyons et al. 1996; Wang et al. 2005). Although the evidence for these interactions is quite compelling, they are not essential for Ste5 function when G $\beta \gamma$ is present, in marked contrast to what we have demonstrated for the PH domain. For example, an allele (cdc244) of Cdc24 that cannot interact with Ste 5 does not exhibit any detectable mating defect (Shimada et al. 2004), and even a null mutation (bem1s) does not eliminate mating (Lyons et al. 1996). In contrast, when Ste5 carries the $\mathrm{PH}$ domain mutations, it is unable to support any detectable mating. Although Ste5 interaction with Bem1 and/or Cdc24 may contribute to Ste5 function when it is at the plasma membrane, our results show that the $\mathrm{PH}$ domain makes an independent and more crucial contribution in the recruitment of Ste5 to the plasma membrane per se.

It has been suggested (Wang and Elion 2003) that a Leu zipper-like sequence (residues 428-455), which resides within the PH domain in Ste5, may mediate its oligomerization. However, all other structurally characterized Leu zippers that form coiled-coils lie in bona fide 4-3 hydrophobic repeats in sequences that adopt an $\alpha$-helical conformation, whereas all of the algorithms we applied indicate that this segment of Ste5 will adopt a $\beta$-strand conformation, more compatible with the $\beta$-sheets found in $\mathrm{PH}$ domains. In this same study, the purported Leu zipper itself was not mutagenized; but, deletion ( $\Delta 474-487$ ) or alteration (L482A L485A) of residues downstream of the proposed Leu zipper, which lie within the C-terminal half of the $\mathrm{PH}$ domain, prevented membrane recruitment and signaling, yet did not interfere with oligomerization, just as we have observed for the R407S K411S mutations near the $\mathrm{N}$-terminal end of the PH domain. However, unlike Ste5(R407S K411S), the $\Delta 474-487$ deletion eliminated, and the L482A L485A mutations greatly reduced, Ste11 binding. Hence, the signaling defect of these alterations was attributed to some as yet undefined role for Ste11 in mediating membrane association of Ste5. However, the R407S K411S mutant, which binds Ste11 normally, clearly separates the role of the $\mathrm{PH}$ domain in membrane binding from Ste11 docking. Also, as we have shown here, wild-type Ste5, but not the PH domain mutant, is recruited to the membrane, even when Ste11 is absent, corroborating a Ste11-independent role for the $\mathrm{PH}$ domain.

The PH domain is a critical mediator of the stable association of Ste 5 with the plasma membrane. In vitro the Ste5 $\mathrm{PH}$ domain binds preferentially to $\operatorname{PtdIns}(4,5) \mathrm{P}_{2}$. Use of fluorescent reporter proteins and other means have shown that PtdIns $(4,5) \mathrm{P}_{2}$ is located predominantly, if not exclusively, in the plasma membrane in S. cerevisiae (Stefan et al. 2002). Thus, the PH domain presumably assists in membrane anchoring of Ste 5 via its direct association with $\operatorname{PtdIns}(4,5) \mathrm{P}_{2}$ in the plasma membrane. Given that the $\mathrm{PH}$ domain is critical for membrane binding, what does interaction of Ste5 with G $\beta \gamma$ contribute? We believe that the most likely explanation for why elimination of the $\mathrm{PH}$ domain ablates the ability of G $\beta \gamma$ to promote membrane recruitment is that a significant aspect of the physiological function of G $\beta \gamma$ binding to Ste5 is not to help anchor Ste5 to the membrane per se, but rather to cause a conformational change that exposes the $\mathrm{PH}$ domain so that it is accessible to interact with the membrane. We have at least some evidence that the $\mathrm{PH}$ domain may be masked in unstimulated Ste5. Only the isolated GST-PH domain bound to phospholipids in vitro; full-length Ste5 (purified from Escherichia coli as a fusion to MalE) did not bind to phospholipids in either the filter overlay or liposome-binding assays (L.S. Garrenton, unpubl.). Therefore, it is important to determine whether G $\beta \gamma$-induced conformational changes and/or oligomerization may regulate the ability of the $\mathrm{PH}$ domain to drive membrane recruitment of Ste5.

\section{Materials and methods}

\section{Yeast strains and media}

Cultivation of strains (Table 1) was at $30^{\circ} \mathrm{C}$ in standard rich (YP) or defined minimal (SC) media (Sherman et al. 1986) containing $2 \%$ glucose (Glc), $2 \%$ raffinose with $0.2 \%$ sucrose (Raf/Suc), or $2 \%$ galactose (Gal) and supplemented with appropriate nutrients to maintain selection for plasmids. Gene induction from the GAL1 promoter was performed as in Inouye et al. (1997a). Solid medium supplemented with $30 \mathrm{mM}$ 3-aminotriazole (3AT) was used, where indicated. Standard yeast genetic techniques were according to Sherman et al. (1986). Mating profi- 
Table 1. S. cerevisiae strains used in this study

\begin{tabular}{|c|c|c|}
\hline Strain & Genotype & Source/reference \\
\hline BYB69 & 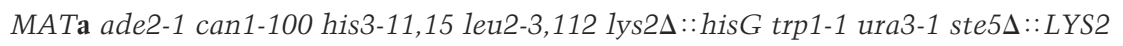 & Inouye et al. $1997 \mathrm{~b}$ \\
\hline BYB84 & MATa gal2 leu2 prb1-1122 pep4-3 prc1-407 trp1 ura3-52 ste5s & Inouye et al. $1997 \mathrm{~b}$ \\
\hline BYB88 & 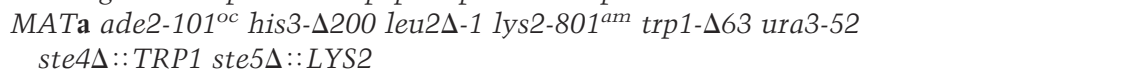 & Inouye et al. $1997 \mathrm{~b}$ \\
\hline YAS1 & BYB84 ste $11 \Delta::$ kanMX6 & A. Saviñon-Tejeda, this laboratory \\
\hline YLG14 ${ }^{\mathrm{a}}$ & BYB84 STE11-3xHA::kanMX6 & This study \\
\hline YLG19 & BYB84 ste $4 \Delta::$ hisG & This study \\
\hline SY1973 & MATa his3-D200 leu2 ura3 trp1 pep4::ura3 FUS1::HIS3 ste5 ::LEU2 STE11-4 & Stevenson et al. 1992 \\
\hline BY4741 & MATa his $3 \Delta 1$ leu2 $2 \Delta 0$ met $15 \Delta 0$ & Research Genetics, Inc. \\
\hline DC17 & $M A T \alpha$ his1 & J.B. Hicks \\
\hline
\end{tabular}

${ }^{a}$ STE11 was C-terminally tagged with the 3xHA epitope via a one-step PCR-based technique for targeted modification of chromosomal loci using pFA6a-3HA-kanMX6 as the template (Longtine et al. 1998).

'STE4 was deleted from the genome using the PCR-based "short flanking homology regions" method with vector pNKY51, containing the hisG::URA3::hisG sequence, as a template (Alani et al. 1987). Correct gene replacement was verified by PCR, and Ura- transformants were then selected on 5-FOA medium.

ciency of MATa cells was assessed as described by Sprague (1991) using strain DC17 as the MAT $\alpha$ tester.

\section{Plasmids and recombinant DNA methods}

Plasmids (Supplementary Table S1) were constructed and propagated in E. coli using standard recombinant DNA methods (Sambrook et al. 1989). Fidelity of all constructs was verified by nucleotide sequence analysis. All PCR utilized Turbo Pfu DNA polymerase (Stratagene). Ste5 $\mathrm{PH}$ domain mutants were generated by site-directed mutagenesis using appropriate mismatch primers.

\section{Purification of GST fusions and overlay assay}

To construct GST-Ste5 PH domain fusions with and without the R407S K411S mutations, an 400-base-pair (bp) fragment (corresponding to Ste5 residues 369-517) was amplified by PCR using pCJ117 and pLG21 as the templates, respectively, and ligated into pGEX-4T (Amersham-Pharmacia), yielding pLG49 and pLG50, respectively. A protease-deficient E. coli strain [BL21-CodonPlus(DE3)-RIL; Stratagene] carrying either pLG49 or pLG50 was grown to $\mathrm{A}_{600 \mathrm{~nm}}=0.2$, and expression of GSTSte5-PH or GST-Ste5-PH(R407S K411S) was induced by addition of isopropyl- $\beta$-D-thiogalacto-pyranoside $(0.4 \mathrm{mM}$ final concentration). After vigorous aeration for $3 \mathrm{~h}$ at room temperature, cells were harvested and GST fusions were purified by column chromatography on glutathione-agarose using standard procedures.

To measure lipid binding, commercially prepared filters with immobilized phospholipids (PIP Strips, Eschelon Biosciences, Inc.) were blocked for $1 \mathrm{~h}$ at room temperature in Odyssey Blocking Solution (Li-Cor). The filters were then incubated at room temperature with purified GST fusions $(0.5 \mu \mathrm{g} / \mathrm{mL})$ in Odyssey Blocking Solution for $3 \mathrm{~h}$, washed three times in Trisbuffered ( $\mathrm{pH} 7.5$ ) saline containing $0.1 \%$ Tween-20, and bound GST fusions were detected by incubation-first for $\sim 1.5 \mathrm{~h}$ with monoclonal $\alpha$-GST antibodies, and then for $60 \mathrm{~min}$ with an appropriate secondary antibody conjugated to an infrared fluorophore-and visualized using an infrared imaging system $/ \mathrm{Od}-$ yssey, Li-Cor).

\section{Binding to liposomes}

Phospholipids and derivatives in chloroform (Avanti Polar Lipids) and Texas Red-DHPE (Molecular Probes) were mixed, evaporated, and hydrated at a final concentration of $1 \mathrm{mg} / \mathrm{mL}$ total phospholipid in HKME buffer $(20 \mathrm{mM}$ HEPES-KOH at $\mathrm{pH}$ 7.0, $160 \mathrm{mM} \mathrm{KOAc,} 1 \mathrm{mM} \mathrm{MgCl}_{2}$, and 0.1 mM EGTA) at room temperature with occasional vortexing. The resulting suspension of multilamellar vesicles was extruded through a polycarbonate filter (400-nm pore size). A sample $(12 \mu \mathrm{L})$ of the resulting unilamellar liposomes was mixed with $48 \mu \mathrm{L}$ HKME containing GST-Ste5-PH or GST-Ste5-PH(R407S K411S) (0.5-5.0 $\mu \mathrm{M})$, and flotation was conducted as described by Matsuoka et al. (1998). Liposome-bound proteins were resolved by SDSPAGE and analyzed by immunoblotting with monoclonal $\alpha$-GST antibodies, as described above. Surface plasmon resonance measurements were made in a device from Reichert Instruments, Inc.

\section{Subcellular localization by fluorescence microscopy}

Transformants expressing GFP fusions were grown, treated with $\alpha$-factor, and prepared for microscopic examination as described previously (Sette et al. 2000). Cells were viewed under a 100× objective using a Delta-Vision Spectris DV4 deconvolution microscope (Applied Precision LCC). Images were collected and processed using API SoftWoRx imaging software and Photoshop (Adobe Systems, Inc.).

\section{Preparation of cell extracts, immunoprecipitation, and immunoblotting}

Yeast cells were grown, harvested, and lysed; cell-free extracts were prepared; and final protein concentration was determined as described previously (Inouye et al. 1997a). For immunoprecipitation, samples (1 mg total protein) were diluted to a final volume of $300 \mu \mathrm{L}$ in L buffer (Inouye et al. 1997a) containing 1\% bovine serum albumin. Subsequent preclearing, immunoprecipitation, washing, and elution were also carried out according to Inouye et al. (1997a), with the exception that the Ste5-3xFlag derivatives were immunoprecipitated using anti-Flag M2 affinity gel (Sigma Chemical Corp.) according to the manufacturer's instructions. The eluted immune complexes, solubilized in SDS-PAGE sample buffer, were boiled for $5 \mathrm{~min}$, clarified by brief centifugation, resolved by $8 \%-12 \%$ SDS-PAGE, and analyzed by immunoblotting. Proteins resolved in slab gels (either cell extract or solubilized immune complexes) were transferred to nitrocellulose, incubated with appropriate primary antibodies, washed, incubated with appropriate secondary antibodies conjugated to infrared fluorophores, and visualized using an in- 
frared imaging system. Ste5 was detected using either affinitypurified polyclonal rabbit IgG (Hasson et al. 1994) or another polyclonal rabbit anti-Ste5 antiserum (gift of Kirsten Benjamin, Molecular Sciences Institute, Berkeley, CA). Other antibodies were as follows: polyclonal rabbit anti-Ste4 (Hirschman et al. 1997), polyclonal goat anti-Ste11 (Santa Cruz Biotechnology, Inc.), mouse monoclonal anti-HA epitope (Covance), mouse monoclonal anti-c-Myc epitope (Evan et al. 1985), mouse monoclonal anti-GFP (Roche Diagnostics, Inc.), mouse monoclonal anti-GST (Sigma Chemical Corp.), Alexa Fluor 680-conjugated goat anti-rabbit IgG and donkey anti-goat IgG (Molecular Probes, Inc.), and IRDye800-conjugated goat anti-mouse IgG and donkey anti-rabbit IgG (Rockland Immunochemicals, Inc.).

\section{Acknowledgments}

We thank D. Ballon, E. Futai, R. Schekman, D. Jenness, M.A. Lemmon, K. Benjamin, and P. Pryciak for advice, reagents, and/ or communication of unpublished results, and T.E. Ryan of Reichert Instruments, Inc., for help with SPR analysis. This work was supported by NIH Predoctoral Traineeships GM07232 (to L.S.G.) and GM07048 (to S.L.Y.), by NCI Predoctoral Traineeship CA09041 (to L.S.G.), by NIH Research Grant GM21841 (to J.T.), and by facilities provided by the Cancer Research Laboratory of the University of California, Berkeley.

\section{References}

Alani, E., Cao, L., and Kleckner, N. 1987. A method for gene disruption that allows repeated use of URA3 selection in the construction of multiply disrupted yeast strains. Genetics 116: $541-545$.

Bateman, A., Coin, L., Durbin, R., Finn, R.D., Hollich, V., Griffiths-Jones, S., Khanna, A., Marshall, M., Moxon, S., Sonnhammer, E.L., et al. 2004. The Pfam protein families database. Nucleic Acids Res. 32: D138-D141.

Butty, A.C., Pryciak, P.M., Huang, L.S., Herskowitz, I., and Peter, M. 1998. The role of Farlp in linking the heterotrimeric $\mathrm{G}$ protein to polarity establishment proteins during yeast mating. Science 282: 1511-1516.

Choi, K.Y., Satterberg, B., Lyons, D.M., and Elion, E.A. 1994. Ste5 tethers multiple protein kinases in the MAP kinase cascade required for mating in S. cerevisiae. Cell 78: 499512.

Cole, G.M. and Reed, S.I. 1991. Pheromone-induced phosphorylation of a $G$ protein $\beta$ subunit in $S$. cerevisiae is associated with an adaptive response to mating pheromone. Cell 64: 703-716.

Drogen, F., O'Rourke, S.M., Stucke, V.M., Jaquenoud, M., Neiman, A.M., and Peter, M. 2000. Phosphorylation of the MEKK Ste11p by the PAK-like kinase Ste20p is required for MAP kinase signaling in vivo. Curr. Biol. 10: 630-639.

Elion, E.A. 2001. The Ste5p scaffold. J. Cell Sci. 114: 3967-3978.

Evan, G.I., Lewis, G.K., Ramsay, G., and Bishop, J.M. 1985. Isolation of monoclonal antibodies specific for human c-myc proto-oncogene product. Mol. Cell. Biol. 5: 3610-3616.

Feng, Y., Song, L.Y., Kincaid, E., Mahanty, S.K., and Elion, E.A. 1998. Functional binding between G $\beta$ and the LIM domain of Ste5 is required to activate the MEKK Ste11. Curr. Biol. 8: 267-278.

Hasson, M.S., Blinder, D., Thorner, J., and Jenness, D.D. 1994. Mutational activation of the STE5 gene product bypasses the requirement for $G$ protein $\beta$ and $\gamma$ subunits in the yeast pheromone response pathway. Mol. Cell. Biol. 14:
1054-1065.

Hirschman, J.E. and Jenness, D.D. 1999. Dual lipid modification of the yeast gy subunit Ste18p determines membrane local-

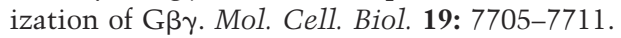

Hirschman, J.E., De Zutter, G.S., Simonds, W.F., and Jenness,

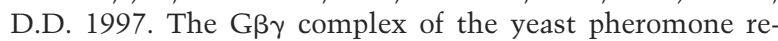
sponse pathway. Subcellular fractionation and protein-protein interactions. J. Biol. Chem. 272: 240-248.

Inouye, C., Dhillon, N., Durfee, T., Zambryski, P.C., and Thorner, J. 1997a. Mutational analysis of STE5 in the yeast Saccharomyces cerevisiae: Application of a differential interaction trap assay for examining protein-protein interactions. Genetics 147: 479-492.

Inouye, C., Dhillon, N., and Thorner, J. 1997b. Ste5 RING-H2 domain: Role in Ste4-promoted oligomerization for yeast pheromone signaling. Science 278: 103-106.

Kelley, L.A., MacCallum, R.M., and Sternberg, M.J. 2000. Enhanced genome annotation using structural profiles in the program 3D-PSSM. J. Mol. Biol. 299: 499-520.

Knaus, M., Wiget, P., Shimada, Y., and Peter, M. 2005. Control of cell polarity in response to intra- and extracellular signals in budding yeast. Novartis Found. Symp. 269: 47-58.

Künzler, M., Trueheart, J., Sette, C., Hurt, E., and Thorner, J. 2001. Mutations in the YRB1 gene encoding yeast ran-binding-protein-1 that impair nucleocytoplasmic transport and suppress yeast mating defects. Genetics 157: 1089-1105.

Lamson, R.E., Winters, M.J., and Pryciak, P.M. 2002. Cdc42 regulation of kinase activity and signaling by the yeast p21activated kinase Ste20. Mol. Cell. Biol. 22: 2939-2951.

Leeuw, T., Fourest-Lieuvin, A., Wu, C., Chenevert, J., Clark, K., Whiteway, M., Thomas, D.Y., and Leberer, E. 1995. Pheromone response in yeast: Association of Bem 1p with proteins of the MAP kinase cascade and actin. Science 270: 1210 1213.

Leeuw, T., Wu, C., Schrag, J.D., Whiteway, M., Thomas, D.Y., and Leberer, E. 1998. Interaction of a G-protein $\beta$-subunit with a conserved sequence in Ste20/PAK family protein kinases. Nature 391: 191-195.

Lemmon, M.A. and Keleti, D. 2005. PH domains. In Modular protein domains (eds. G. Cesareni et al.), pp. 337-363. WileyVCH Verlag GmbH \& Co., Weinheim, Germany.

Lin, K., Simossis, V.A., Taylor, W.R., and Heringa, J. 2005. A simple and fast secondary structure prediction method using hidden neural networks. Bioinformatics 21: 152-159.

Longtine, M.S., McKenzie III, A., Demarini, D.J., Shah, N.G., Wach, A., Brachat, A., Philippsen, P., and Pringle, J.R. 1998. Additional modules for versatile and economical PCR-based gene deletion and modification in Saccharomyces cerevisiae. Yeast 14: 953-961.

Lyons, D.M., Mahanty, S.K., Choi, K.Y., Manandhar, M., and Elion, E.A. 1996. The SH3-domain protein Bem1 coordinates mitogen-activated protein kinase cascade activation with cell cycle control in Saccharomyces cerevisiae. Mol. Cell. Biol. 16: 4095-4106.

Mahanty, S.K., Wang, Y., Farley, F.W., and Elion, E.A. 1999. Nuclear shuttling of yeast scaffold Ste5 is required for its recruitment to the plasma membrane and activation of the mating MAPK cascade. Cell 98: 501-512.

Marchler-Bauer, A., Anderson, J.B., DeWeese-Scott, C., Fedorova, N.D., Geer, L.Y., He, S., Hurwitz, D.I., Jackson, J.D., Jacobs, A.R., Lanczycki, C.J., et al. 2003. CDD: A curated Entrez database of conserved domain alignments. Nucleic Acids Res. 31: 383-387.

Marcus, A., Polverino, A., Barr, M., and Wigler, M. 1994. Complexes between STE5 and components of the pheromoneresponsive mitogen-activated protein kinase module. Proc. 
Nat1. Acad. Sci. 91: 7762-7766.

Maru, Y., Afar, D.E., Witte, O.N., and Shibuya, M. 1996. The dimerization property of glutathione S-transferase partially reactivates Bcr-Abl lacking the oligomerization domain. $J$. Biol. Chem. 271: 15353-15357.

Matsuoka, K., Orci, L., Amherdt, M., Bednarek, S.Y., Hamamoto, S., Schekman, R., and Yeung, T. 1998. COPII-coated vesicle formation reconstituted with purified coat proteins and chemically defined liposomes. Cell 93: 263-275.

McGuffin, L.J., Bryson, K., and Jones, D.T. 2000. The PSIPRED protein structure prediction server. Bioinformatics 16: 404405.

Mitchell, D.A. and Deschenes, R.J. 1995. Characterization of protein prenylation in Saccharomyces cerevisiae. Methods Enzymol. 250: 68-78.

Ohya, Y., Qadota, H., Anraku, Y., Pringle, J.R., and Botstein, D. 1993. Suppression of yeast geranylgeranyl transferase I defect by alternative prenylation of two target GTPases, Rholp and Cdc42p. Mol. Biol. Cell 4: 1017-1025.

Pollastri, G., Przybylski, D., Rost, B., and Baldi, P. 2002. Improving the prediction of protein secondary structure in three and eight classes using recurrent neural networks and profiles. Proteins 47: 228-235.

Printen, J.A. and Sprague Jr., G.F. 1994. Protein-protein interactions in the yeast pheromone response pathway: Ste5p interacts with all members of the MAP kinase cascade. Genetics 138: 609-619.

Pryciak, P.M. and Huntress, F.A. 1998. Membrane recruitment of the kinase cascade scaffold protein Ste5 by the G $\beta \gamma$ complex underlies activation of the yeast pheromone response pathway. Genes \& Dev. 12: 2684-2697.

Sambrook, J., Fritsch, E.F., and Maniatis, T. 1989. Molecular cloning: A laboratory manual. Cold Spring Harbor Laboratory, Cold Spring Harbor, NY.

Sato, T.K., Overduin, M., and Emr, S.D. 2001. Location, location, location: Membrane targeting directed by PX domains. Science 294: 1881-1885.

Sette, C., Inouye, C.J., Stroschein, S.L., Iaquinta, P.J., and Thorner, J. 2000. Mutational analysis suggests that activation of the yeast pheromone response mitogen-activated protein kinase pathway involves conformational changes in the Ste5 scaffold protein. Mol. Biol. Cell 11: 4033-4049.

Sherman, F., Fink, G.R., and Hicks, J.B. 1986. Laboratory course manual for methods in yeast genetics. Cold Spring Harbor Laboratory, Cold Spring Harbor, NY.

Shimada, Y., Wiget, P., Gulli, M.P., Bi, E., and Peter, M. 2004. The nucleotide exchange factor Cdc24p may be regulated by auto-inhibition. EMBO J. 23: 1051-1062.

Sprague Jr., G.F. 1991. Assay of yeast mating reaction. Methods Enzymol. 194: 77-93.

Stefan, C.J., Audhya, A., and Emr, S.D. 2002. The yeast synaptojanin-like proteins control the cellular distribution of phosphatidylinositol (4,5)-bisphosphate. Mol. Biol. Cell 13: 542-557.

Stevenson, B.J., Rhodes, N., Errede, B., and Sprague Jr., G.F. 1992. Constitutive mutants of the protein kinase STE11 activate the yeast pheromone response pathway in the absence of the G protein. Genes \& Dev. 6: 1293-1304.

Wang, Y. and Dohlman, H.G. 2004. Pheromone signaling mechanisms in yeast: A prototypical sex machine. Science 306: $1508-1509$.

Wang, Y. and Elion, E.A. 2003. Nuclear export and plasma membrane recruitment of the Ste5 scaffold are coordinated with oligomerization and association with signal transduction components. Mol. Biol. Cell 14: 2543-2558.

Wang, Y., Chen, W., Simpson, D.M., and Elion, E.A. 2005.
Cdc24 regulates nuclear shuttling and recruitment of the Ste5 scaffold to a heterotrimeric G protein in S. cerevisiae. I. Biol. Chem. 280: 13084-13096.

Whiteway, M.S., Wu, C., Leeuw, T., Clark, K., Fourest-Lieuvin, A., Thomas, D.Y., and Leberer, E. 1995. Association of the yeast pheromone response $G$ protein $\beta \gamma$ subunits with the MAP kinase scaffold Ste5p. Science 269: 1572-1575.

Wiget, P., Shimada, Y., Butty, A.C., Bi, E., and Peter, M. 2004. Site-specific regulation of the GEF Cdc24p by the scaffold protein Farlp during yeast mating. EMBO J. 23: 1063-1074.

Wild, A.C., Yu, J.W., Lemmon, M.A., and Blumer, K.J. 2004. The p21-activated protein kinase-related kinase Cla4 is a coincidence detector of signaling by Cdc42 and phosphatidylinositol 4-phosphate. J. Biol. Chem. 279: 17101-17110.

Winters, M.J., Lamson, R.E., Nakanishi, H., Neiman, A.M., and Pryciak, P.M. 2005. A membrane binding domain in the ste5 scaffold synergizes with $g \beta \gamma$ binding to control localization and signaling in pheromone response. Mol. Cell 20: 21-32.

Yablonski, D., Marbach, I., and Levitzki, A. 1996. Dimerization of Ste5, a mitogen-activated protein kinase cascade scaffold protein, is required for signal transduction. Proc. Natl. Acad. Sci. 93: 13864-13869.

Yu, J.W., Mendrola, J.M., Audhya, A., Singh, S., Keleti, D., DeWald, D.B., Murray, D., Emr, S.D., and Lemmon, M.A. 2004. Genome-wide analysis of membrane targeting by $S$. cerevisiae pleckstrin homology domains. Mol. Cell 13: 677-688. 


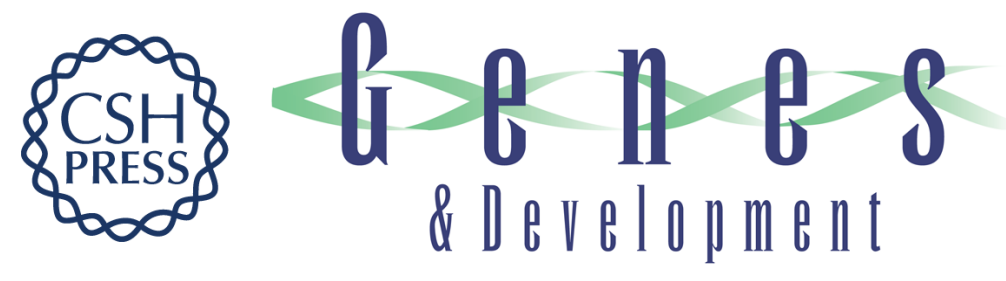

\section{Function of the MAPK scaffold protein, Ste5, requires a cryptic PH domain}

Lindsay S. Garrenton, Susan L. Young and Jeremy Thorner

Genes Dev. 2006, 20:

Access the most recent version at doi:10.1101/gad.1413706

Supplemental http://genesdev.cshlp.org/content/suppl/2006/06/30/20.14.1946.DC1
Material

References This article cites 49 articles, 29 of which can be accessed free at: http://genesdev.cshlp.org/content/20/14/1946.full.html\#ref-list-1

License

Email Alerting

Receive free email alerts when new articles cite this article - sign up in the box at the top Service right corner of the article or click here.

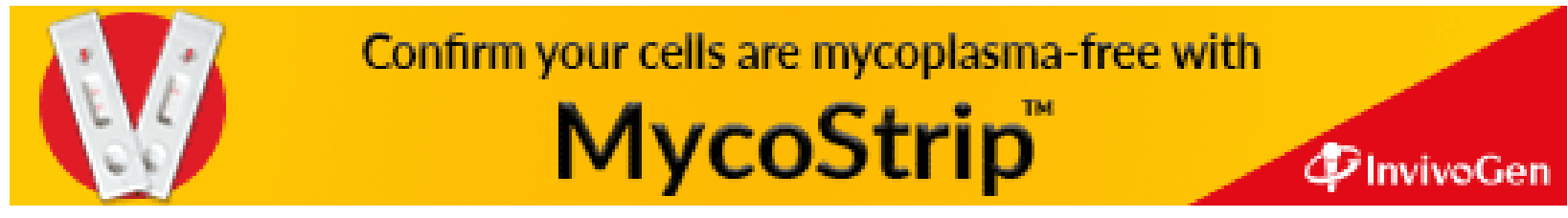

